\title{
Mycobacterial Interspersed Repetitive Unit-variable Number Tandem Repeat (MIRU-VNTR) Typing Lacks Discriminatory Power in the Genetic Analysis of Bovine Tuberculosis in Egypt
}

\author{
Hebatallah Ahmed Mahgoub ${ }^{1, *}$, Mohamed F. Salama ${ }^{2}$, Mohamed El-Adl ${ }^{2}$, Ahmed Ammar $^{3}$, \\ Ali Amer ${ }^{4}$, Samar Atwa ${ }^{5}$, Youssef Alseady ${ }^{6}$, Walaa Awadin', Emad Mokhtar ${ }^{4}$ \\ ${ }^{1}$ Pathology Department, Faculty of Veterinary Medicine, Mansoura University, Egypt \\ ${ }^{2}$ Department of Biochemistry and Chemistry of Nutrition, Faculty of Veterinary Medicine, Mansoura University, Egypt \\ ${ }^{3}$ Department of Bacteriology, Mycology and Immunology, Faculty of Veterinary Medicine, Zagazig University, Egypt \\ ${ }^{4}$ Tuberculosis Department, Animal Health Research Centre, Egypt \\ ${ }^{5}$ Department of Internal Medicine, Infectious and Fish Diseases, Faculty of Veterinary Medicine, Mansoura University, Egypt \\ ${ }^{6}$ Physiology Department, Faculty of Veterinary Medicine, Mansoura University, Egypt \\ *Corresponding author: hebamahgoub@mans.edu.eg, heba.a.mahgoub@gmail.com
}

\begin{abstract}
Tuberculosis (TB) is one of the most important infectious zoonotic diseases of vertebrates worldwide. TB in animals is primarily known from cases in cattle and other bovids for which the disease is generally referred to as bovine TB. The major causative agent of bovine TB is Mycobacterium bovis (M. bovis), a member of the Mycobacterium tuberculosis complex. Animal TB is a disease of high economic relevance within the context of livestock farming as it directly affects animal productivity and also influences international trade of animal products. In this study, we aimed at mycobacterial interspersed repetitive unit-variable number tandem repeat (MIRU-VNTR) analysis of cases of TB infection in local cattle in Egypt. Therefore, various samples (milk and blood samples) were collected from cattle farms (in Damietta Province) that were positive for tuberculin test. Mycobacterial isolation was tested on milk samples, but it showed negative result. DNA was extracted from blood samples. Five mycobacterial genes (IS6110, katG, gyrA, oxyR, pncA) were used for further confirmation of field TB infection. All blood samples were positive for Mycobacterial-specific genes. Twelve MIRU-VNTR loci were used to test their discriminatory power in the genetic analysis of TB, but such MIRU-VNTR loci typing failed to show any discriminatory power for the genetic analysis of bovine TB cases.
\end{abstract}

Keywords: bovine tuberculosis, MIRU-VNTR typing, tuberculin test

Cite This Article: Hebatallah Mahgoub, Mohamed F. Salama, Mohamed El-Adl, Ahmed Ammar, Ali Amer, Samar Atwa, Youssef Alseady, Walaa Awadin, and Emad Mokhtar, "Mycobacterial Interspersed Repetitive Unit-variable Number Tandem Repeat (MIRU-VNTR) Typing Lacks Discriminatory Power in the Genetic Analysis of Bovine Tuberculosis in Egypt." American Journal of Microbiological Research, vol. 5, no. 6 (2017): 142-147. doi: 10.12691/ajmr-5-6-5.

\section{Introduction}

Tuberculosis (TB) in animals is primarily known from cases in cattle and other bovids for which the disease is generally referred to as bovine $\mathrm{TB}[1,2]$. The major causative agent of bovine TB is Mycobacterium bovis (M. bovis), a member of the Mycobacterium tuberculosis complex. Animal TB is a disease of high economic relevance within the context of livestock farming as it directly affects animal productivity and also influences international trade of animal products [3]. M. bovis infections have also been detected in wildlife and can have severe consequences for the ecosystem. Moreover, animal TB bears a zoonotic potential and is therefore of public health concern. Therefore, diagnosis of bovine TB is crucial for a proper control of disease transmission to animals and humans. To achieve that, a thorough knowledge of the available strains of $M$. bovis is required.

The identification of $M$. bovis by conventional bacteriological techniques is time consuming. Therefore, molecular identification techniques (spoligotyping and mycobacterial interspersed repetitive unit-variable number tandem repeat (MIRU-VNTR) analysis) have been developed and were proved to be quicker and reliable in discriminating M. bovis from the other Mycobacterium tuberculosis complex [4-11]. Repeated sequences were identified in the genome of $M$. bovis and M. tuberculosis and were used to type M. bovis by MIRU-VNTR analysis [4-11]. Therefore, we aimed at MIRU-VNTR analysis of cases of TB infection in local cattle in Egypt. Here, we applied MIRU-VNTR typing on samples collected from tuberculin-positive cattle in local farms in Damietta Province. 


\section{Materials and Methods}

\subsection{Sample Collection}

Samples were collected from different cattle farms, in Damietta Province. Samples were obtained from cattle that showed positive results for tuberculin test. Sample collection was performed under the recommendations and approval of Mansoura University Animal Care and Use Committee.

\subsubsection{Milk Samples}

Fifty milk samples were collected from animals (in two cattle farms in Damietta Province) that showed positive results for tuberculin test and had mastitis. Five ml of milk were collected aseptically in sterile tubes.

\subsubsection{Blood Samples}

One hundred blood samples were collected from cattle (in three different farms in Damietta Province) that showed positive results for tuberculin test.

\subsection{Isolation of Mycobacterium Species on Culture Medium}

\subsubsection{Preparation of Culture Media Used for Isolation (Middlebrook 7H10 Agar (BBL, Difco))}

Nineteen grams of the agar powder were suspended in $900 \mathrm{ml}$ of purified water containing $5 \mathrm{ml}$ of glycerol and mixed thoroughly. The mixture was heated with frequent agitation and boiled for one minute to completely dissolve the powder. The mixture was autoclaved and then $100 \mathrm{ml}$ of Middlebrook OADC Enrichment were added to the medium when cooled to $50-55^{\circ} \mathrm{C}$.

\subsubsection{Preparation of Milk Samples}

Sample preparation was performed according to a previously published procedure [12]. Two $\mathrm{ml}$ of $4 \% \mathrm{H}_{2} \mathrm{SO}_{4}$ were added to the sample and was incubated for $30 \mathrm{~min}$ at room temperature. Later, $16 \mathrm{ml}$ of sterile distilled water were added to the previous mixture, and the whole mixture was centrifuged at $3000 \mathrm{rpm}$ for $20 \mathrm{~min}$. The supernatant was decanted into 5\% phenol, and the sediment was used to make a direct smear, which was inoculated into two L-J medium slant (one contains $4 \%$ sodium pyruvate, and the other contains $5 \%$ glycerol) and was then incubated at $37^{\circ} \mathrm{C}$ for 3 weeks. Cultures were examined daily for one week and then once weekly for 6-8 weeks.

\subsection{DNA Extraction from Blood Samples}

DNA preparation from blood was performed by using DNA preparation Kit following the protocol of the manufacturer (Jenabioscience, Germany).

\subsection{Confirmation of Mycobacterial Infection by Specific Gene Analysis}

DNA, from blood samples collected from tuberculin-positive cattle farms, was tested for IS6110, katG, gyrA, oxyR, pncA genes (Table 1). Primer sequences for target genes were selected from previous literature [13] and were obtained from Metabion (Germany).

\subsection{MIRU-VNTR Typing}

MIRU-VNTR typing of DNA samples was performed using the primers shown in Table 1. Primer sequences for target loci were selected from previous reports $[4,5,6]$. Basically, the $20 \mu \mathrm{l}$ PCR reaction consisted of $10 \mu \mathrm{lpf}$ PCR mastermix (Intron Biotechnology, Korea), $1 \mu \mathrm{l}$ (10 pmol) of each primer (forward or reverse), $2 \mu$ l of sample DNA and up to $20 \mu \mathrm{l}$ nuclease-free water. The thermal cycling included 1 cycle of $12 \mathrm{~min}$ at $94^{\circ} \mathrm{C}, 40$ cycles of $30 \mathrm{sec}$ at $94^{\circ} \mathrm{C}, 1 \mathrm{~min}$ at the chosen annealing temperature according to the melting temperature of each primer sets (Table 1), $90 \mathrm{sec}$ at $72^{\circ} \mathrm{C}$, followed by 1 cycle of $7 \mathrm{~min}$ at $72^{\circ} \mathrm{C}$. PCR products were resolved by electrophoresis using $1.5 \%$ agarose gels (in TAE buffer, Bioshop). The gel was placed in an electrophoresis tank containing $0.5 \% \mathrm{TBE}$ and run for $1 \mathrm{~h}$ at $80 \mathrm{~V}$. Markers (50 and $100 \mathrm{bp}$ ladder, Qiagen and iNtTRON, respectively) were electrophoresed beside the DNA samples to measure the size of the DNA fragments. The DNA fragments were then visualised on a UV transilluminator using long wavelength ultraviolet light.

Table 1. Primer sequences, the expected product size for each used primer-set, and the appropriate annealing temperature used for PCR (Metabion) $[4,5,6,13]$

\begin{tabular}{|c|c|c|c|c|}
\hline Target locus & Forward primer sequence (5'-3') & Reverse primer sequence (5'-3') & $\begin{array}{c}\text { Ecpected Locus/ } \\
\text { Repeat unit size (bp) }\end{array}$ & $\begin{array}{c}\text { Annealing } \\
\text { temperature }\left({ }^{\circ} \mathrm{C}\right)\end{array}$ \\
\hline ETR-A & AAATCGGTCCCATCACCTTCTTAT & CGAAGCCTGGGGTGCCCGCGATTT & 75 & 60 \\
\hline ETR-B & GCGAACACCAGGACAGCATCATG & GGCATGCCGGTGATCGAGTGG & 57 & 60 \\
\hline ETR-C & GTGAGTCGCTGCAGAACCTGCAG & GGCGTCTTGACCTCCACGAGTG & 58 & 60 \\
\hline ETR-D & CAGGTCACAACGAGAGGAAGAGC & GCGGATCGGCCAGCGACTCCTC & 77 & 60 \\
\hline ETR-E & GCGGATCGGCCAGCGACTCCTC & CGGAACGCTGGTCACCACCTAAG & 53 & 60 \\
\hline MIRU-10 & GTTCTTGACCAACTGCAGTCGTCC & GCCACCTTGGTGATCAGCTACCT & 53 & 58.8 \\
\hline MIRU-16 & TCGGTGATCGGGTCCAGTCCAAGTA & CCCGTCGTGCAGCCCTGGTAC & 53 & 58.8 \\
\hline MIRU-26 & TAGGTCTACCGTCGAAATCTGTGAC & CATAGGCGACCAGGCGAATAG & 51 & 58.8 \\
\hline MIRU-40 & GGGTTGCTGGATGACAACGTGT & GGGTGATCTCGGCGAAATCAGATA & 54 & 58.8 \\
\hline QUB-3232 & CAGACCCGGCGTCATCAAC & CCAAGGGCGGCATTGTGTT & 56 & 55 \\
\hline QUB-3336 & ATCCCCGCGGTACCCATC & GCCAGCGGTGTCGACTATCC & 59 & 55 \\
\hline QUB-1895 & GGTGCACGGCCTCGGCTCC & AAGCCCCGCCGCCAATCAA & 57 & 55 \\
\hline IS6110 & CTCGTCCAGCGCCGCTTCGG & CCTGCGAGCGTAGGCGTCGG & 123 & 68 \\
\hline katG & TCAGCCACGACCTCGTCGG & AGGCGGATGCGACCACCGTT & 163 & 65 \\
\hline gyrA & CGAGACCATGGGCAACTACCA & CATTGCCTGGCGAGCCGAA & 131 & 65 \\
\hline oxyR & CGCGCTGTCAGAGCTGACTTT & TCTGCGGAATCAGTGTCACC & 150 & 60 \\
\hline pncA & ATCAGCGACTACCTGGCCGA & GATTGCCGACGTGTCCAGAC & 180 & 66 \\
\hline
\end{tabular}




\subsection{Data Analysis}

Allele frequency for the single MIRU-VNTR locus was calculated as previously stated [14], where the genetic profile or the frequency of each assigned copy number (number of certain copy number appearance in the selected population) was divided by the number of samples/isolates in the selected population. Furthermore, genetic diversity for the locus or allele diversity $(h)$ was calculated as $h=1-\Sigma x i^{2}[n /(n-1)]$, where $x i$ is the frequency of the $i$ th allele at the locus, $\Sigma x i^{2}$ is the summation of all recorded (allele frequency) ${ }^{2}$ for a single locus, $n$ is the number of isolates in the sample, and $n /(n-1)$ is a correction for bias in small samples [14].

\section{Results}

\subsection{Mycobacterial Isolation}

Milk samples (collected from farms that showed positive results for tuberculin test) did not show any positive result for mycobacterial isolation.

Table 2. The recorded copy number analysis of tested MIRU-VNTR loci in all tested samples and the results of specific gene analysis

\begin{tabular}{|c|c|c|c|c|c|c|c|c|c|c|c|c|c|c|c|c|c|c|}
\hline $\begin{array}{l}\stackrel{0}{0} \\
\text { ठ․ }\end{array}$ & $\begin{array}{l}\stackrel{0}{\tilde{\Xi}} \\
\text { हैँ }\end{array}$ & $\begin{array}{l}\stackrel{\nabla}{\star} \\
\vdots\end{array}$ & 心 & $\underset{b_{0}}{\pi}$ & 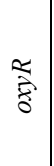 & $\begin{array}{l}\mathbb{Z} \\
\Xi\end{array}$ & 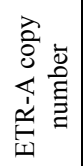 & 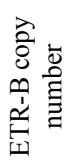 & 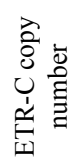 & 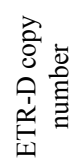 & 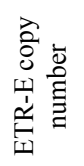 & 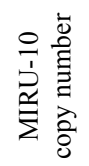 & 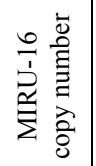 & 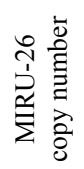 & 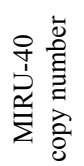 & 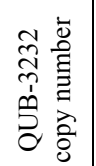 & 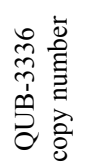 & 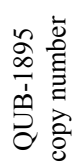 \\
\hline \multirow{40}{*}{$\mathrm{Fa}$} & & + & + & + & + & + & $5 \times 1$ & $\mathrm{x} 1$ & 1 & $\mathrm{x} 1$ & $\times 2$ & $\mathrm{x} 1$ & $\mathrm{x} 1$ & $\mathrm{x} 1$ & $+\times 1$ & $6 \times 2$ & $9 \times 2$ & $7 \times 2$ \\
\hline & Blood 2 & + & + & + & . & . & $75 \times 1$ & $57 \times 1$ & $58 \times 1$ & $77 \times 1$ & $3 \times 2$ & $53 \times 1$ & $53 \times 1$ & $1 \times 1$ & $4 \times 1$ & $56 \times 2$ & $9 \times 2$ & $7 \times 2$ \\
\hline & Blood 3 & + & + & + & + & + & $75 \times 1$ & $57 \times 1$ & $58 \times 1$ & $77 \times 1$ & $53 \times 2$ & $53 \times 1$ & $53 \times 1$ & $1 \times 1$ & $54 \times 1$ & $66 \times 2$ & $9 \times 2$ & $7 \times 2$ \\
\hline & Blood 4 & + & + & + & + & + & $75 \times 1$ & $57 \times 1$ & $58 \times 1$ & $77 \times 1$ & $53 \times 2$ & $53 \times 1$ & $53 \times 1$ & $51 \times 1$ & $54 \times 1$ & $56 \times 2$ & $59 \times 2$ & $57 \times 2$ \\
\hline & Blood 5 & + & + & + & + & +1 & $75 \times 1$ & $57 \times 1$ & $58 \times 1$ & $77 \times 1$ & $53 \times 2$ & $53 \times 1$ & $53 \times 1$ & $51 \times 1$ & $54 \times 1$ & $56 \times 2$ & $59 \times 2$ & $57 \times 2$ \\
\hline & Blood 6 & + & + & + & + & + & $75 \times 1$ & $57 \times 1$ & $58 \times 1$ & $77 \times 1$ & $53 \times 2$ & $53 \times 1$ & $53 \times 1$ & $1 \times 1$ & $54 \times 1$ & $56 \times 2$ & $9 \times 2$ & $57 \times 2$ \\
\hline & Blood 7 & + & + & + & + & r & $75 \times 1$ & $57 \times 1$ & $8 \times 1$ & $77 \times 1$ & $53 \times 2$ & $53 \times 1$ & $53 \times 1$ & $1 \times 1$ & $4 \times 1$ & $6 \times 2$ & $9 \times 2$ & $7 \times 2$ \\
\hline & Blood 8 & + & + & + & + & + & $75 \times 1$ & $57 \times 1$ & $\mathrm{x} 1$ & $77 \times 1$ & $3 \times 2$ & $3 \times 1$ & $3 \times 1$ & $\mathrm{x} 1$ & $4 \times 1$ & $6 \times 2$ & $9 \times 2$ & $7 \times 2$ \\
\hline & Blood 9 & + & + & + & + & + & $75 \times 1$ & $57 \times 1$ & $8 \times 1$ & $77 \times 1$ & $53 \times 2$ & $53 \times 1$ & $53 \times 1$ & $1 \times 1$ & $54 \times 1$ & $56 \times 2$ & $9 \times 2$ & $57 \times 2$ \\
\hline & Blood 10 & + & + & + & + & +1 & $75 \times 1$ & $57 \times 1$ & $88 \times 1$ & $77 \times 1$ & $53 \times 2$ & $53 \times 1$ & $53 \times 1$ & $1 \times 1$ & $54 \times 1$ & $56 \times 2$ & $59 \times 2$ & $57 \times 2$ \\
\hline & Blood 11 & + & + & + & + & 1 & $75 \times 1$ & $57 \times 1$ & $8 \times 1$ & $77 \times 1$ & $53 \times 2$ & $53 \times 1$ & $3 \times 1$ & $1 \times 1$ & $4 \times 1$ & $6 \times 2$ & $9 \times 2$ & $57 \times 2$ \\
\hline & Bloc & + & + & + & + & +1 & $75 \times 1$ & $57 \times 1$ & $x 1$ & $77 \times 1$ & $53 \times 2$ & $3 \times 1$ & $3 \times 1$ & $1 \times 1$ & $4 \times 1$ & $6 \times 2$ & $9 \times 2$ & $7 \times 2$ \\
\hline & Blood 13 & + & + & + & + & + & $75 \times 1$ & $57 \times 1$ & $58 \times 1$ & $77 \times 1$ & $53 \times 2$ & $53 \times 1$ & $53 \times 1$ & $1 \times 1$ & $54 \times 1$ & $56 \times 2$ & $9 \times 2$ & $57 \times 2$ \\
\hline & Blood 14 & + & + & + & + & 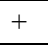 & $75 \times 1$ & $57 \times 1$ & $58 \times 1$ & $77 \times 1$ & $53 \times 2$ & $53 \times 1$ & $53 \times 1$ & $51 \times 1$ & $54 \times 1$ & $56 \times 2$ & $59 \times 2$ & $57 \times 2$ \\
\hline & Blood 15 & + & + & + & + & + & $75 \times 1$ & $57 \times 1$ & $8 \times 1$ & $77 \times 1$ & $53 \times 2$ & $53 \times 1$ & $53 \times 1$ & $1 \times 1$ & $54 \times 1$ & $56 \times 2$ & $9 \times 2$ & $57 \times 2$ \\
\hline & 6 & + & 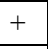 & 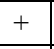 & 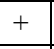 & + & $75 \times 1$ & $57 \times 1$ & $3 \times 1$ & $77 \times 1$ & $53 \times 2$ & $3 \times 1$ & $3 \times 1$ & $1 \times 1$ & $4 \times 1$ & $5 \times 2$ & $9 \times 2$ & $7 \times 2$ \\
\hline & Blood 17 & + & + & + & 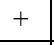 & 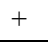 & $75 \times 1$ & $57 \times 1$ & $8 \times 1$ & $77 \times 1$ & $53 \times 2$ & $53 \times 1$ & $53 \times 1$ & $1 \times 1$ & $4 \times 1$ & $6 \times 2$ & $9 \times 2$ & $7 \times 2$ \\
\hline & Blood 18 & + & + & + & + & + & $75 \times 1$ & $57 \times 1$ & $58 \times 1$ & $77 \times 1$ & $53 \times 2$ & $53 \times 1$ & $53 \times 1$ & $51 \times 1$ & $54 \times 1$ & $56 \times 2$ & $59 \times 2$ & $57 \times 2$ \\
\hline & Blood 19 & + & + & + & + & + & $75 \times 1$ & $57 \times 1$ & $58 \times 1$ & $77 \times 1$ & $53 \times 2$ & $53 \times 1$ & $53 \times 1$ & $1 \times 1$ & $4 \times 1$ & $56 \times 2$ & $9 \times 2$ & $57 \times 2$ \\
\hline & & + & + & + & + & + & $75 \times 1$ & $57 \times 1$ & $\mathrm{x} 1$ & $77 \times 1$ & $3 \times 2$ & $\mathrm{x} 1$ & $\mathrm{x} 1$ & $\mathrm{x} 1$ & $\mathrm{x} 1$ & $\mathrm{x} 2$ & $9 \times 2$ & $7 \times 2$ \\
\hline & Blood 21 & + & + & + & 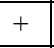 & 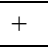 & $75 \times 1$ & $57 \times 1$ & $\mathrm{x} 1$ & $77 \times 1$ & $53 \times 2$ & $53 \times 1$ & $3 \times 1$ & $1 \times 1$ & $4 \times 1$ & $6 \times 2$ & $9 \times 2$ & $7 \times 2$ \\
\hline & Blood 22 & + & + & + & + & + & $75 \times 1$ & $57 \times 1$ & $58 \times 1$ & $77 \times 1$ & $53 \times 2$ & $53 \times 1$ & $53 \times 1$ & $1 \times 1$ & $54 \times 1$ & $56 \times 2$ & $59 \times 2$ & $57 \times 2$ \\
\hline & Blood 23 & + & + & + & + & 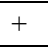 & $75 \times 1$ & $57 \times 1$ & $8 \times 1$ & $77 \times 1$ & $53 \times 2$ & $53 \times 1$ & $53 \times 1$ & $1 \times 1$ & $4 \times 1$ & $56 \times 2$ & $59 \times 2$ & $57 \times 2$ \\
\hline & & + & + & + & 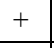 & 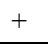 & $75 \times 1$ & $57 \times 1$ & $\mathrm{x} 1$ & 1 & $3 \times 2$ & x 1 & x 1 & $\mathrm{x} 1$ & $\times 1$ & 2 & $9 \times 2$ & 2 \\
\hline & Blood 25 & + & + & + & + & + & $75 \times 1$ & $57 \times 1$ & $58 \times 1$ & $77 \times 1$ & $53 \times 2$ & $53 \times 1$ & $53 \times 1$ & $1 \times 1$ & $54 \times 1$ & $56 \times 2$ & $9 \times 2$ & $57 \times 2$ \\
\hline & Blood 26 & + & + & + & + & + & $75 \times 1$ & $57 \times 1$ & $58 \times 1$ & $77 \times 1$ & $53 \times 2$ & $53 \times 1$ & $53 \times 1$ & $51 \times 1$ & $54 \times 1$ & $56 \times 2$ & $59 \times 2$ & $57 \times 2$ \\
\hline & Blood 27 & + & + & + & + & + & $75 \times 1$ & $57 \times 1$ & $58 \times 1$ & $77 \times 1$ & $53 \times 2$ & $53 \times 1$ & $53 \times 1$ & $1 \times 1$ & $54 \times 1$ & $56 \times 2$ & $59 \times 2$ & $57 \times 2$ \\
\hline & 20 & + & + & + & 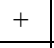 & 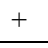 & $75 \times 1$ & $57 \times 1$ & $8 \times 1$ & $77 \times 1$ & $53 \times 2$ & $53 \times 1$ & $\mathrm{x} 1$ & $1 \times 1$ & $4 \times 1$ & $6 \times 2$ & $59 \times 2$ & $7 \times 2$ \\
\hline & 29 & + & + & + & + & + & $75 \times 1$ & $57 \times 1$ & $8 \times 1$ & $77 \times 1$ & $53 \times 2$ & $53 \times 1$ & $53 \times 1$ & $1 \times 1$ & $54 \times 1$ & $56 \times 2$ & $9 \times 2$ & $7 \times 2$ \\
\hline & Blood 30 & + & + & + & + & + & $75 \times 1$ & $57 \times 1$ & $58 \times 1$ & $77 \times 1$ & $53 \times 2$ & $53 \times 1$ & $53 \times 1$ & $51 \times 1$ & $54 \times 1$ & $56 \times 2$ & $9 \times 2$ & $57 \times 2$ \\
\hline & Blood 31 & + & + & + & + & + & $75 \times 1$ & $57 \times 1$ & $8 \times 1$ & $77 \times 1$ & $53 \times 2$ & $53 \times 1$ & $53 \times 1$ & $1 \times 1$ & $4 \times 1$ & $6 \times 2$ & $9 \times 2$ & $57 \times 2$ \\
\hline & Blood 32 & + & + & + & + & + & $75 \times 1$ & $57 \times 1$ & $8 \times 1$ & $77 \times 1$ & $53 \times 2$ & $53 \times 1$ & $53 \times 1$ & $1 \times 1$ & $4 \times 1$ & $56 \times 2$ & $59 \times 2$ & $7 \times 2$ \\
\hline & d 33 & + & + & + & + & 4 & $75 \times 1$ & $57 \times 1$ & $\mathrm{x} 1$ & $77 \times 1$ & $53 \times 2$ & $\mathrm{x} 1$ & $3 \times 1$ & $\mathrm{x} 1$ & $\mathrm{x} 1$ & $x 2$ & $9 \times 2$ & $7 \times 2$ \\
\hline & $\mathrm{Bl}$ & + & + & + & + & + & $75 \times 1$ & $57 \times 1$ & $\mathrm{x} 1$ & $77 \times 1$ & $3 \times 2$ & $\mathrm{x} 1$ & $\mathrm{x} 1$ & $\mathrm{x} 1$ & $\mathrm{x} 1$ & $\times 2$ & $\times 2$ & $7 \times 2$ \\
\hline & $\mathrm{Blo}$ & + & + & + & + & + & $75 \times 1$ & $57 \times 1$ & $\mathrm{x} 1$ & $77 \times 1$ & $53 \times 2$ & $53 \times 1$ & $3 \times 1$ & $\mathrm{x} 1$ & $x 1$ & $6 \times 2$ & $9 \times 2$ & $57 \times 2$ \\
\hline & Blood 36 & + & + & + & + & + & $75 \times 1$ & $57 \times 1$ & $58 \times 1$ & $77 \times 1$ & $53 \times 2$ & $53 \times 1$ & $53 \times 1$ & $51 \times 1$ & $54 \times 1$ & $56 \times 2$ & $59 \times 2$ & $57 \times 2$ \\
\hline & Blood 37 & + & + & + & + & + & $75 \times 1$ & $57 \times 1$ & $58 \times 1$ & $77 \times 1$ & $53 \times 2$ & $53 \times 1$ & $53 \times 1$ & $51 \times 1$ & $54 \times 1$ & $56 \times 2$ & $59 \times 2$ & $57 \times 2$ \\
\hline & 38 & + & + & + & + & + & $75 \times 1$ & $57 \times 1$ & $\mathrm{x} 1$ & $77 \times 1$ & $\mathrm{x} 2$ & $\mathrm{x} 1$ & $\mathrm{x} 1$ & $\mathrm{x} 1$ & $\mathrm{x} 1$ & $5 \times 2$ & $\times 2$ & $7 \times 2$ \\
\hline & d 39 & + & + & + & + & + & $75 \times 1$ & $57 \times 1$ & $\mathrm{x} 1$ & $77 \times 1$ & $\times 2$ & $53 \times 1$ & $\mathrm{x} 1$ & $\mathrm{x} 1$ & $\mathrm{x} 1$ & $\mathrm{x} 2$ & $\mathrm{x} 2$ & $57 \times 2$ \\
\hline & Blood 40 & + & + & + & + & + & $75 \times 1$ & $57 \times 1$ & $58 \times 1$ & $77 \times 1$ & $53 \times 2$ & $53 \times 1$ & $53 \times 1$ & $51 \times 1$ & $54 \times 1$ & $56 \times 2$ & $59 \times 2$ & $57 \times 2$ \\
\hline
\end{tabular}




\begin{tabular}{|c|c|c|c|c|c|c|c|c|c|c|c|c|c|c|c|c|c|c|}
\hline $\begin{array}{l}0 \\
\stackrel{0}{0} \\
\stackrel{0}{n}\end{array}$ & 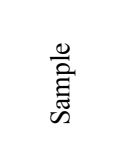 & $\begin{array}{l}\stackrel{i}{6} \\
\vdots \\
\vdots\end{array}$ & $\stackrel{\Xi}{\Xi}$ & $\overrightarrow{\sigma_{0}}$ & 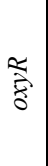 & 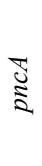 & 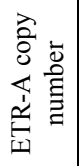 & 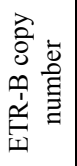 & 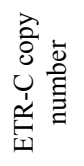 & 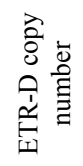 & 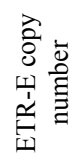 & 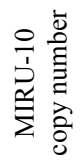 & 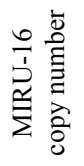 & 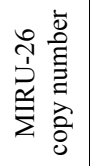 & 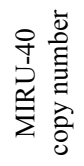 & 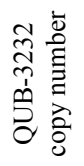 & 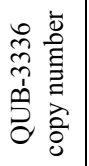 & 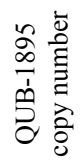 \\
\hline \multirow{30}{*}{ Farm B } & Blood 41 & + & + & + & + & + & $75 \times 1$ & $57 \times 1$ & $58 \times 1$ & $77 \times 1$ & $53 \times 2$ & $53 \times 1$ & $53 \times 1$ & $51 \times 1$ & $54 \times 1$ & $56 \times 2$ & $59 \times 2$ & $57 \times 2$ \\
\hline & Blood 42 & + & + & + & + & + & $75 \times 1$ & $57 \times 1$ & $58 \times 1$ & $77 \times 1$ & $53 \times 2$ & $53 \times 1$ & $53 \times 1$ & $51 \times 1$ & $54 \times 1$ & $56 \times 2$ & $59 \times 2$ & $57 \times 2$ \\
\hline & Blood 43 & + & + & + & + & + & $75 \times 1$ & $57 \times 1$ & $58 \times 1$ & $77 \times 1$ & $53 \times 2$ & $53 \times 1$ & $53 \times 1$ & $51 \times 1$ & $54 \times 1$ & $56 \times 2$ & $59 \times 2$ & $57 \times 2$ \\
\hline & Blood 44 & + & + & + & + & + & $75 \times 1$ & $57 \times 1$ & $58 \times 1$ & $77 \times 1$ & $53 \times 2$ & $53 \times 1$ & $53 \times 1$ & $51 \times 1$ & $54 \times 1$ & $56 \times 2$ & $59 \times 2$ & $57 \times 2$ \\
\hline & Blood 45 & + & + & + & + & + & $75 \times 1$ & $57 \times 1$ & $58 \times 1$ & $77 \times 1$ & $53 \times 2$ & $53 \times 1$ & $53 \times 1$ & $51 \times 1$ & $54 \times 1$ & $56 \times 2$ & $59 \times 2$ & $57 \times 2$ \\
\hline & Blood 46 & + & + & + & + & + & $75 \times 1$ & $57 \times 1$ & $58 \times 1$ & $77 \times 1$ & $53 \times 2$ & $53 \times 1$ & $53 \times 1$ & $51 \times 1$ & $54 \times 1$ & $56 \times 2$ & $59 \times 2$ & $57 \times 2$ \\
\hline & Blood 47 & + & + & + & + & + & $75 \times 1$ & $57 \times 1$ & $58 \times 1$ & $77 \times 1$ & $53 \times 2$ & $53 \times 1$ & $53 \times 1$ & $51 \times 1$ & $54 \times 1$ & $56 \times 2$ & $59 \times 2$ & $57 \times 2$ \\
\hline & Blood 48 & + & + & + & + & + & $75 \times 1$ & $57 \times 1$ & $58 \times 1$ & $77 \times 1$ & $53 \times 2$ & $53 \times 1$ & $53 \times 1$ & $51 \times 1$ & $54 \times 1$ & $56 \times 2$ & $59 \times 2$ & $57 \times 2$ \\
\hline & Blood 49 & + & + & + & + & + & $75 \times 1$ & $57 \times 1$ & $58 \times 1$ & $77 \times 1$ & $53 \times 2$ & $53 \times 1$ & $53 \times 1$ & $51 \times 1$ & $54 \times 1$ & $56 \times 2$ & $59 \times 2$ & $57 \times 2$ \\
\hline & Blood 50 & + & + & + & + & + & $75 \times 1$ & $57 \times 1$ & $58 \times 1$ & $77 \times 1$ & $53 \times 2$ & $53 \times 1$ & $53 \times 1$ & $51 \times 1$ & $54 \times 1$ & $56 \times 2$ & $59 \times 2$ & $57 \times 2$ \\
\hline & Blood 51 & + & + & + & + & + & $75 \times 1$ & $57 \times 1$ & $58 \times 1$ & $77 \times 1$ & $53 \times 2$ & $53 \times 1$ & $53 \times 1$ & $51 \times 1$ & $54 \times 1$ & $56 \times 2$ & $59 \times 2$ & $57 \times 2$ \\
\hline & Blood 52 & + & + & + & + & + & $75 \times 1$ & $57 \times 1$ & $58 \times 1$ & $77 \times 1$ & $53 \times 2$ & $53 \times 1$ & $53 \times 1$ & $51 \times 1$ & $54 \times 1$ & $56 \times 2$ & $59 \times 2$ & $57 \times 2$ \\
\hline & Blood 53 & + & + & + & + & + & $75 \times 1$ & $57 \times 1$ & $58 \times 1$ & $77 \times 1$ & $53 \times 2$ & $53 \times 1$ & $53 \times 1$ & $51 \times 1$ & $54 \times 1$ & $56 \times 2$ & $59 \times 2$ & $57 \times 2$ \\
\hline & Blood 54 & + & + & + & + & + & $75 \times 1$ & $57 \times 1$ & $58 \times 1$ & $77 \times 1$ & $53 \times 2$ & $53 \times 1$ & $53 \times 1$ & $51 \times 1$ & $54 \times 1$ & $56 \times 2$ & $59 \times 2$ & $57 \times 2$ \\
\hline & Blood 55 & + & + & + & + & + & $75 \times 1$ & $57 \times 1$ & $58 \times 1$ & $77 \times 1$ & $53 \times 2$ & $53 \times 1$ & $53 \times 1$ & $51 \times 1$ & $54 \times 1$ & $56 \times 2$ & $59 \times 2$ & $57 \times 2$ \\
\hline & Blood 56 & + & + & + & + & + & $75 \times 1$ & $57 \times 1$ & $58 \times 1$ & $77 \times 1$ & $53 \times 2$ & $53 \times 1$ & $53 \times 1$ & $51 \times 1$ & $54 \times 1$ & $56 \times 2$ & $59 \times 2$ & $57 \times 2$ \\
\hline & Blood 57 & + & + & + & + & + & $75 \times 1$ & $57 \times 1$ & $58 \times 1$ & $77 \times 1$ & $53 \times 2$ & $53 \times 1$ & $53 \times 1$ & $51 \times 1$ & $54 \times 1$ & $56 \times 2$ & $59 \times 2$ & $57 \times 2$ \\
\hline & Blood 58 & + & + & + & + & + & $75 \times 1$ & $57 \times 1$ & $58 \times 1$ & $77 \times 1$ & $53 \times 2$ & $53 \times 1$ & $53 \times 1$ & $51 \times 1$ & $54 \times 1$ & $56 \times 2$ & $59 \times 2$ & $57 \times 2$ \\
\hline & Blood 59 & + & + & + & + & + & $75 \times 1$ & $57 \times 1$ & $58 \times 1$ & $77 \times 1$ & $53 \times 2$ & $53 \times 1$ & $53 \times 1$ & $51 \times 1$ & $54 \times 1$ & $56 \times 2$ & $59 \times 2$ & $57 \times 2$ \\
\hline & Blood 60 & + & + & + & + & + & $75 \times 1$ & $57 \times 1$ & $58 \times 1$ & $77 \times 1$ & $53 \times 2$ & $53 \times 1$ & $53 \times 1$ & $51 \times 1$ & $54 \times 1$ & $56 \times 2$ & $59 \times 2$ & $57 \times 2$ \\
\hline & Blood 61 & + & + & + & + & + & $75 \times 1$ & $57 \times 1$ & $58 \times 1$ & $77 \times 1$ & $53 \times 2$ & $53 \times 1$ & $53 \times 1$ & $51 \times 1$ & $54 \times 1$ & $56 \times 2$ & $59 \times 2$ & $57 \times 2$ \\
\hline & Blood 62 & + & + & + & + & + & $75 \times 1$ & $57 \times 1$ & $58 \times 1$ & $77 \times 1$ & $53 \times 2$ & $53 \times 1$ & $53 \times 1$ & $51 \times 1$ & $54 \times 1$ & $56 \times 2$ & $59 \times 2$ & $57 \times 2$ \\
\hline & Blood 63 & + & + & + & + & + & $75 \times 1$ & $57 \times 1$ & $58 \times 1$ & $77 \times 1$ & $53 \times 2$ & $53 \times 1$ & $53 \times 1$ & $51 \times 1$ & $54 \times 1$ & $56 \times 2$ & $59 \times 2$ & $57 \times 2$ \\
\hline & Blood 64 & + & + & + & + & + & $75 \times 1$ & $57 \times 1$ & $58 \times 1$ & $77 \times 1$ & $53 \times 2$ & $53 \times 1$ & $53 \times 1$ & $51 \times 1$ & $54 \times 1$ & $56 \times 2$ & $59 \times 2$ & $57 \times 2$ \\
\hline & Blood 65 & + & + & + & + & + & $75 \times 1$ & $57 \times 1$ & $58 \times 1$ & $77 \times 1$ & $53 \times 2$ & $53 \times 1$ & $53 \times 1$ & $51 \times 1$ & $54 \times 1$ & $56 \times 2$ & $59 \times 2$ & $57 \times 2$ \\
\hline & Blood 66 & + & + & + & + & + & $75 \times 1$ & $57 \times 1$ & $58 \times 1$ & $77 \times 1$ & $53 \times 2$ & $53 \times 1$ & $53 \times 1$ & $51 \times 1$ & $54 \times 1$ & $56 \times 2$ & $59 \times 2$ & $57 \times 2$ \\
\hline & Blood 67 & + & + & + & + & + & $75 \times 1$ & $57 \times 1$ & $58 \times 1$ & $77 \times 1$ & $53 \times 2$ & $53 \times 1$ & $53 \times 1$ & $51 \times 1$ & $54 \times 1$ & $56 \times 2$ & $59 \times 2$ & $57 \times 2$ \\
\hline & Blood 68 & + & + & + & + & + & $75 \times 1$ & $57 \times 1$ & $58 \times 1$ & $77 \times 1$ & $53 \times 2$ & $53 \times 1$ & $53 \times 1$ & $51 \times 1$ & $54 \times 1$ & $56 \times 2$ & $59 \times 2$ & $57 \times 2$ \\
\hline & Blood 69 & + & + & + & + & + & $75 \times 1$ & $57 \times 1$ & $58 \times 1$ & $77 \times 1$ & $53 \times 2$ & $53 \times 1$ & $53 \times 1$ & $51 \times 1$ & $54 \times 1$ & $56 \times 2$ & $59 \times 2$ & $57 \times 2$ \\
\hline & Blood 70 & + & + & + & + & + & $75 \times 1$ & $57 \times 1$ & $58 \times 1$ & $77 \times 1$ & $53 \times 2$ & $53 \times 1$ & $53 \times 1$ & $51 \times 1$ & $54 \times 1$ & $56 \times 2$ & $59 \times 2$ & $57 \times 2$ \\
\hline \multirow{30}{*}{ Farm C } & Blood 71 & + & + & + & + & + & $75 \times 1$ & $57 \times 1$ & $58 \times 1$ & $77 \times 1$ & $53 \times 2$ & $53 \times 1$ & $53 \times 1$ & $51 \times 1$ & $54 \times 1$ & $56 \times 2$ & $59 \times 2$ & $57 \times 2$ \\
\hline & Blood 72 & + & + & + & + & + & $75 \times 1$ & $57 \times 1$ & $58 \times 1$ & $77 \times 1$ & $53 \times 2$ & $53 \times 1$ & $53 \times 1$ & $51 \times 1$ & $54 \times 1$ & $56 \times 2$ & $59 \times 2$ & $57 \times 2$ \\
\hline & Blood 73 & + & + & + & + & + & $75 \times 1$ & $57 \times 1$ & $58 \times 1$ & $77 \times 1$ & $53 \times 2$ & $53 \times 1$ & $53 \times 1$ & $51 \times 1$ & $54 \times 1$ & $56 \times 2$ & $59 \times 2$ & $57 \times 2$ \\
\hline & Blood 74 & + & + & + & + & + & $75 \times 1$ & $57 \times 1$ & $58 \times 1$ & $77 \times 1$ & $53 \times 2$ & $53 \times 1$ & $53 \times 1$ & $51 \times 1$ & $54 \times 1$ & $56 \times 2$ & $59 \times 2$ & $57 \times 2$ \\
\hline & Blood 75 & + & + & + & + & + & $75 \times 1$ & $57 \times 1$ & $58 \times 1$ & $77 \times 1$ & $53 \times 2$ & $53 \times 1$ & $53 \times 1$ & $51 \times 1$ & $54 \times 1$ & $56 \times 2$ & $59 \times 2$ & $57 \times 2$ \\
\hline & Blood 76 & + & + & + & + & + & $75 \times 1$ & $57 \times 1$ & $58 \times 1$ & $77 \times 1$ & $53 \times 2$ & $53 \times 1$ & $53 \times 1$ & $51 \times 1$ & $54 \times 1$ & $56 \times 2$ & $59 \times 2$ & $57 \times 2$ \\
\hline & Blood 77 & + & + & + & + & + & $75 \times 1$ & $57 \times 1$ & $58 \times 1$ & $77 \times 1$ & $53 \times 2$ & $53 \times 1$ & $53 \times 1$ & $51 \times 1$ & $54 \times 1$ & $56 \times 2$ & $59 \times 2$ & $57 \times 2$ \\
\hline & Blood 78 & + & + & + & + & + & $75 \times 1$ & $57 \times 1$ & $58 \times 1$ & $77 \times 1$ & $53 \times 2$ & $53 \times 1$ & $53 \times 1$ & $51 \times 1$ & $54 \times 1$ & $56 \times 2$ & $59 \times 2$ & $57 \times 2$ \\
\hline & Blood 79 & + & + & + & + & + & $75 \times 1$ & $57 \times 1$ & $58 \times 1$ & $77 \times 1$ & $53 \times 2$ & $53 \times 1$ & $53 \times 1$ & $51 \times 1$ & $54 \times 1$ & $56 \times 2$ & $59 \times 2$ & $57 \times 2$ \\
\hline & Blood 80 & + & + & + & + & + & $75 \times 1$ & $57 \times 1$ & $58 \times 1$ & $77 \times 1$ & $53 \times 2$ & $53 \times 1$ & $53 \times 1$ & $51 \times 1$ & $54 \times 1$ & $56 \times 2$ & $59 \times 2$ & $57 \times 2$ \\
\hline & Blood 81 & + & + & + & + & + & $75 \times 1$ & $57 \times 1$ & $58 \times 1$ & $77 \times 1$ & $53 \times 2$ & $53 \times 1$ & $53 \times 1$ & $51 \times 1$ & $54 \times 1$ & $56 \times 2$ & $59 \times 2$ & $57 \times 2$ \\
\hline & Blood 82 & + & + & + & + & + & $75 \times 1$ & $57 \times 1$ & $58 \times 1$ & $77 \times 1$ & $53 \times 2$ & $53 \times 1$ & $53 \times 1$ & $51 \times 1$ & $54 \times 1$ & $56 \times 2$ & $59 \times 2$ & $57 \times 2$ \\
\hline & Blood 83 & + & + & + & + & + & $75 \times 1$ & $57 \times 1$ & $58 \times 1$ & $77 \times 1$ & $53 \times 2$ & $53 \times 1$ & $53 \times 1$ & $51 \times 1$ & $54 \times 1$ & $56 \times 2$ & $59 \times 2$ & $57 \times 2$ \\
\hline & Blood 84 & + & + & + & + & + & $75 \times 1$ & $57 \times 1$ & $58 \times 1$ & $77 \times 1$ & $53 \times 2$ & $53 \times 1$ & $53 \times 1$ & $51 \times 1$ & $54 \times 1$ & $56 \times 2$ & $59 \times 2$ & $57 \times 2$ \\
\hline & Blood 85 & + & + & + & + & + & $75 \times 1$ & $57 \times 1$ & $58 \times 1$ & $77 \times 1$ & $53 \times 2$ & $53 \times 1$ & $53 \times 1$ & $51 \times 1$ & $54 \times 1$ & $56 \times 2$ & $59 \times 2$ & $57 \times 2$ \\
\hline & Blood 86 & + & + & + & + & + & $75 \times 1$ & $57 \times 1$ & $58 \times 1$ & $77 \times 1$ & $53 \times 2$ & $53 \times 1$ & $53 \times 1$ & $51 \times 1$ & $54 \times 1$ & $56 \times 2$ & $59 \times 2$ & $57 \times 2$ \\
\hline & Blood 87 & + & + & + & + & + & $75 \times 1$ & $57 \times 1$ & $58 \times 1$ & $77 \times 1$ & $53 \times 2$ & $53 \times 1$ & $53 \times 1$ & $51 \times 1$ & $54 \times 1$ & $56 \times 2$ & $59 \times 2$ & $57 \times 2$ \\
\hline & Blood 88 & + & + & + & + & + & $75 \times 1$ & $57 \times 1$ & $58 \times 1$ & $77 \times 1$ & $53 \times 2$ & $53 \times 1$ & $53 \times 1$ & $51 \times 1$ & $54 \times 1$ & $56 \times 2$ & $59 \times 2$ & $57 \times 2$ \\
\hline & Blood 89 & + & + & + & + & + & $75 \times 1$ & $57 \times 1$ & $58 \times 1$ & $77 \times 1$ & $53 \times 2$ & $53 \times 1$ & $53 \times 1$ & $51 \times 1$ & $54 \times 1$ & $56 \times 2$ & $59 \times 2$ & $57 \times 2$ \\
\hline & Blood 90 & + & + & + & + & + & $75 \times 1$ & $57 \times 1$ & $58 \times 1$ & $77 \times 1$ & $53 \times 2$ & $53 \times 1$ & $53 \times 1$ & $51 \times 1$ & $54 \times 1$ & $56 \times 2$ & $59 \times 2$ & $57 \times 2$ \\
\hline & Blood 91 & + & + & + & + & + & $75 \times 1$ & $57 \times 1$ & $58 \times 1$ & $77 \times 1$ & $53 \times 2$ & $53 \times 1$ & $53 \times 1$ & $51 \times 1$ & $54 \times 1$ & $56 \times 2$ & $59 \times 2$ & $57 \times 2$ \\
\hline & Blood 92 & + & + & + & + & + & $75 \times 1$ & $57 \times 1$ & $58 \times 1$ & $77 \times 1$ & $53 \times 2$ & $53 \times 1$ & $53 \times 1$ & $51 \times 1$ & $54 \times 1$ & $56 \times 2$ & $59 \times 2$ & $57 \times 2$ \\
\hline & Blood 93 & + & + & + & + & + & $75 \times 1$ & $57 \times 1$ & $58 \times 1$ & $77 \times 1$ & $53 \times 2$ & $53 \times 1$ & $53 \times 1$ & $51 \times 1$ & $54 \times 1$ & $56 \times 2$ & $59 \times 2$ & $57 \times 2$ \\
\hline & Blood 94 & + & + & + & + & + & $75 \times 1$ & $57 \times 1$ & $58 \times 1$ & $77 \times 1$ & $53 \times 2$ & $53 \times 1$ & $53 \times 1$ & $51 \times 1$ & $54 \times 1$ & $56 \times 2$ & $59 \times 2$ & $57 \times 2$ \\
\hline & Blood 95 & + & + & + & + & + & $75 \times 1$ & $57 \times 1$ & $58 \times 1$ & $77 \times 1$ & $53 \times 2$ & $53 \times 1$ & $53 \times 1$ & $51 \times 1$ & $54 \times 1$ & $56 \times 2$ & $59 \times 2$ & $57 \times 2$ \\
\hline & Blood 96 & + & + & + & + & + & $75 \times 1$ & $57 \times 1$ & $58 \times 1$ & $77 \times 1$ & $53 \times 2$ & $53 \times 1$ & $53 \times 1$ & $51 \times 1$ & $54 \times 1$ & $56 \times 2$ & $59 \times 2$ & $57 \times 2$ \\
\hline & Blood 97 & + & + & + & + & + & $75 \times 1$ & $57 \times 1$ & $58 \times 1$ & $77 \times 1$ & $53 \times 2$ & $53 \times 1$ & $53 \times 1$ & $51 \times 1$ & $54 \times 1$ & $56 \times 2$ & $59 \times 2$ & $57 \times 2$ \\
\hline & Blood 98 & + & + & + & + & + & $75 \times 1$ & $57 \times 1$ & $58 \times 1$ & $77 \times 1$ & $53 \times 2$ & $53 \times 1$ & $53 \times 1$ & $51 \times 1$ & $54 \times 1$ & $56 \times 2$ & $59 \times 2$ & $57 \times 2$ \\
\hline & Blood 99 & + & + & + & + & + & $75 \times 1$ & $57 \times 1$ & $58 \times 1$ & $77 \times 1$ & $53 \times 2$ & $53 \times 1$ & $53 \times 1$ & $51 \times 1$ & $54 \times 1$ & $56 \times 2$ & $59 \times 2$ & $57 \times 2$ \\
\hline & Blood 100 & + & + & + & + & + & $75 \times 1$ & $57 \times 1$ & $58 \times 1$ & $77 \times 1$ & $53 \times 2$ & $53 \times 1$ & $53 \times 1$ & $51 \times 1$ & $54 \times 1$ & $56 \times 2$ & $59 \times 2$ & $57 \times 2$ \\
\hline
\end{tabular}




\subsection{Confirmation of Mycobacterial Infection by Specific Gene Analysis}

DNA, extracted from blood samples (collected from tuberculin-positive cattle farms), was tested for IS6110, $k a t G, \operatorname{gyr} A, \operatorname{oxy} R$, pncA genes. All DNA samples were positive for all tested genes, and gave the expected PCR product sizes (Table 2).

\subsection{MIRU-VNTR Typing}

Although the selected MIRU-VNTR loci gave positive PCR result for their relative copy number (Table 1), none of the selected MIRU-VNTR loci showed polymorphism (in the copy number) across all studied samples.

\subsection{Allelic Diversity of Target MIRU-VNTR Loci}

The allelic diversity was calculated for selected MIRU-VNTR loci (Table 3). Due to the lack of polymorphism (in the MIRU-VNTR copy number), none of the selected MIRU-VNTR loci showed any discriminatory power in the genetic analysis of field samples.

Table 3. Calculating Allele frequency and genetic diversity (h) for each tested locus

\begin{tabular}{|l|c|c|c|c|}
\hline $\begin{array}{c}\text { Tandem } \\
\text { Repeat locus }\end{array}$ & $\begin{array}{c}\text { Number of } \\
\text { alleles }\end{array}$ & $\begin{array}{c}\text { Copy } \\
\text { number }\end{array}$ & $\begin{array}{c}\text { Allele } \\
\text { Frequency }\end{array}$ & $\begin{array}{c}\text { Allele } \\
\text { diversity (h) }\end{array}$ \\
\hline ETR-A & 1 & 1 & 1 & 0 \\
\hline ETR-B & 1 & 1 & 1 & 0 \\
\hline ETR-C & 1 & 1 & 1 & 0 \\
\hline ETR-D & 1 & 1 & 1 & 0 \\
\hline ETR-E & 1 & 2 & 1 & 0 \\
\hline MIRU-10 & 1 & 1 & 1 & 0 \\
\hline MIRU-16 & 1 & 1 & 1 & 0 \\
\hline MIRU-26 & 1 & 1 & 1 & 0 \\
\hline MIRU-40 & 1 & 1 & 1 & 0 \\
\hline QUB-3232 & 1 & 2 & 1 & 0 \\
\hline QUB-3336 & 1 & 2 & 1 & 0 \\
\hline QUB-1895 & 1 & 2 & 1 & 0 \\
\hline
\end{tabular}

\section{Discussion}

MIRU-VNTR is used as an approach to discriminate Mycobacterium tuberculosis complex [4-11]. In this study, we have accomplished MIRU-VNTR analysis in cases of bovine TB in Egypt, along with mycobacterial specific gene analysis (IS6110, katG, gyrA, oxyR, pncA).

Mycobacterial isolation failed from collected milk samples from positive cases of tuberculin test. Milk was previously recorded as a poor carrier for bovine TB infection, where researchers obtained poor percentage (10\%) for bovine TB isolation from positive or suspected cases of TB infection [15].

All tested DNA samples, extracted from blood samples (collected from tuberculin-positive cattle farms), were positive for the selected mycobacterial genes, IS6110, $k a t G, \operatorname{gyr} A, \operatorname{oxy} R, p n c A$, at the expected product size. A previous study performed on M. bovis in Germany [13], revealed the positivity of some $M$. bovis-positive, IS6110-positive samples to katG, gyrA, oxyR, and pncA. However, in the same study, other $M$. bovis-positive, IS6110-positive samples were only positive for gyrA. In the same study, M. tuberculosis samples lacked one, two, or three of the four investigated targets ( kat $G, \operatorname{gyr} A$, oxyR, and $p n c A$ ), and only one sample contained the four targets.

The MIRU-VNTR loci analysis did not show any discriminatory power for bovine TB genetic analysis. Our findings were close to researchers in New Zealand who worked on $M$. bovis MIRU-VNTR typing [11], where MIRU-10, MIRU-16, MIRU-40, QUB-3232, QUB-3336, and QUB-1895 did not have any discriminatory power for Mycobacteria typing, and ETR-C and ETR-D had medium and high discriminatory power for Mycobacteria typing, respectively. However, in their study, ETR-B and ETR-A had medium and high discriminatory powers for Mycobacteria typing, respectively, and ETR-E did not have any discriminatory power for Mycobacteria typing. Our study findings were also close to the data revealed by Korean researchers who worked on M. bovis MIRU-VNTR typing, where MIRU-10, MIRU-16, MIRU-40, and ETR-C had no discriminatory power for bovine TB typing in Korea [6]. In their study, MIRU-26, QUB-1895, and ETR-B had minimal discriminatory power for bovine TB typing. On the other hand, QUB-3232 and ETR-A had medium discriminatory power, and QUB-3336 had the highest discriminatory power across all tested loci. Furthermore, MIRU-VNTR typing of bovine TB in Brazil, caused by M. bovis, revealed the high discriminatory power of ETR-A, ETR-B, ETR-C, and MIRU-16, when compared to the minimal to nil discriminatory power of MIRU-26, MIRU-40, and MIRU-10 [7]. The lack of discriminatory power in our result could be attributed to the small geographical area our investigation included.

In comparison with molecular typing of Mycobacterium tuberculosis, some ETRs were found polymorphic in Mycobacterium tuberculosis strains by other researchers in Thailand [9], where high discriminatory power for human TB typing was recorded by using ETR-B, ETR-D, and ETR-E. In contrast to our findings, where MIRUs did not have any discriminatory power, their work showed another high discriminatory power for TB typing by using MIRU-10, MIRU-16, and MIRU-26.

\section{Conclusion}

In the current study, we were able to perform MIRUVNTR loci analysis for TB-infected cattle in Egypt. However, the MIRU-VNTR loci analysis did not any discriminatory power for bovine TB genetic analysis. Therefore, further analysis is required for clear distinction between field isolates/samples, either through using other MIRU-VNTR loci or through spligotyping.

\section{Acknowledgments}

The project was funded by Mansoura University, Egypt. 


\section{Conflict of Interest}

The authors declare that they have no conflict of interest.

\section{Author Contribution}

Project design was performed by HM and MS. Sample collection was achieved by HM, SA, YA, and WA. Microbial isolation was performed by collaboration between A. Ammar, A. Amer, and EM. MIRU-VNTR typing was performed by collaboration between HM, MS, and ME. Mycobacterial gene-specific analysis and project data analysis were accomplished by HM.

\section{References}

[1] Michel, A.L., Muller, B., van Helden, P.D. Mycobacterium bovis at the animal-human interface: a problem, or not? Vet Microbiol 2010; 140(3-4): 371-81.

[2] Coscolla, M., Gagneux, S. Consequences of genomic diversity in Mycobacterium tuberculosis. Semin Immunol 2014; 26(6):431-44.

[3] Ramos, D.F., Tavares, L., da Silva, P.E., Dellagostin, O.A Molecular typing of Mycobacterium bovis isolates: a review. Braz J Microbiol 2014; 45(2):365-72.

[4] Skuce, R.A., McCorry, T.P., McCarroll, J.F., Roring, S.M., Scott, A.N., Brittain, D., et al. Discrimination of Mycobacterium tuberculosis complex bacteria using novel VNTR-PCR targets. Microbiology 2002; 148(Pt 2): 519-28.

[5] Supply, P., Allix, C., Lesjean, S., Cardoso-Oelemann, M., Rusch-Gerdes, S., Willery, E., et al. Proposal for standardization of optimized mycobacterial interspersed repetitive unit-variable-number tandem repeat typing of Mycobacterium tuberculosis. J Clin Microbiol 2006; 44(12):4498-510.

[6] Jeon, B., Je, S., Park, J., Kim, Y., Lee, E.G., Lee, H., et al. Variable number tandem repeat analysis of Mycobacterium bovis isolates from Gyeonggi-do, Korea. J Vet Sci 2008; 9(2):145-53.

[7] Carvalho, R.C., Vasconcellos, S.E., Issa Mde, A., Soares Filho, P.M., Mota, P.M., Araujo, F.R., et al. Molecular Typing of Mycobacterium bovis from Cattle Reared in Midwest Brazil. PloS one 2016; 11(9):e0162459.

[8] Le Fleche, P., Fabre, M., Denoeud, F., Koeck, J.L., Vergnaud, G. High resolution, on-line identification of strains from the Mycobacterium tuberculosis complex based on tandem repeat typing. BMC Microbiol 2002; 2:37.

[9] Smittipat, N., Billamas, P., Palittapongarnpim, M., Thong-On, A., Temu, M.M., Thanakijcharoen, P., et al. Polymorphism of variable-number tandem repeats at multiple loci in Mycobacterium tuberculosis. J Clin Microbiol 2005; 43(10):5034-43.

[10] Roring, S., Scott, A., Brittain, D., Walker, I., Hewinson, G., Neill, S., et al. Development of variable-number tandem repeat typing of Mycobacterium bovis: comparison of results with those obtained by using existing exact tandem repeats and spoligotyping. J Clin Microbiol 2002; 40(6):2126-33.

[11] Price-Carter, M., Rooker, S., Collins, D.M. Comparison of 45 variable number tandem repeat (VNTR) and two direct repeat (DR) assays to restriction endonuclease analysis for typing isolates of Mycobacterium bovis. Vet Microbiol 2011; 150(1-2):107-14.

[12] Marks, J. Ending the routine guinea-pig test. Tubercle 1972; 53(1):31-4.

[13] Zink, A.R., Nerlich, A.G. Molecular strain identification of the Mycobacterium tuberculosis complex in archival tissue samples. J Clin Pathol 2004; 57(11):1185-92.

[14] Selander, R.K., Caugant, D.A., Ochman, H., Musser, J.M., Gilmour, M.N., Whittam, T.S. Methods of multilocus enzyme electrophoresis for bacterial population genetics and systematics. Appl Environ Microbiol 1986; 51(5):873-84.

[15] Prado, R.B., Nangoti, H., Mendonca, L.J.P., Chi, K.D. Isolation of Mycobacterium spp. in milk from cows suspected or positive to tuberculosis. Braz J Vet Res An Sci 2001; 38(6):284-7. 